factors in carcinogenicity. Samples of asbestos extracted with organic solvents have produced as many tumours as the original samples, so it now seems unlikely that contaminating hydrocarbons or trace metals are required for the induction of mesothelioma or bronchogenic carcinoma by asbestos.

\section{HYDROGEN BONDING \\ (HF) 2 Revealed}

from a Correspondent

IMPORTANT progress has been made in the past year or so in understanding the simplest hydrogen bond in the gas phase, that in the hydrogen fluoride dimer $(\mathrm{HF})_{2}$. This may well prove to be the breakthrough which leads to a clarification of other cases.

It would be difficult to exaggerate the importance of hydrogen bonds. Without their special stability water would remain a gas down to low temperatures and almost all models in biophysics would be quite untenable. There is already a very large literature on the hydrogen bond, but real steps forward have proved elusive. This has been at least partly because intermolecular hydrogen bonding favours the liquid and solid states, whereas the gas phase is usually to be preferred when it comes to elucidating electronic and molecular structures - each molecule is then unfettered by interaction with its neighbours.

First there were the theoretical $a b$ initio calculations of Kollman and Allen (J. Chem. Phys., 52, $5085 ;$ 1970) and of Del Bene and Pople (J. Chem. Phys., 55, 2296; 1971). Both groups agreed that in the equilibrium arrangement the two HF molecules remained as identifiable units with normal bond lengths. They agreed, too, that the hydrogen bond section $\mathbf{F}-\mathbf{H} \ldots \mathrm{F}$ is almost linear, with a distance between fluorine atoms of about $270 \mathrm{pm}(2.70 \AA)$ and with the remaining hydrogen atom offset from this line. They disagree, however, about the exact size of the angle between the linear section and the second $\mathrm{F}-\mathrm{H}$ bond. J. A. Pople in his recent Centenary Lecture to the Chemical Society confirmed that extended calculations support his earlier value of about $111^{\circ}$. The stabilization energy of the dimer is calculated as about $25 \mathrm{~kJ} \mathrm{~mol}^{-1}$ and this agrees with such experimental evidence as there is. Surprisingly the hydrogen atom in the bond is slightly denuded of electrons compared with a hydrogen atom in a monomeric molecule of HF; correspondingly, the fluorine is slightly more negatively charged and is thus able to be on the receiving side of a further hydrogen bond so that stable chains and rings can be formed by aggregates of three or more molecules. For calculations whose input is only the value of the atomic constants and the atomic number of fluorine the detail and reliability are impressive.

But most scientists place greater trust in experimental evidence and this has been provided even more recently. The difficulty with the $(\mathrm{HF})_{2}$ dimer is that it normally coexists with polymers of the form (HF) ${ }_{n}$, where $n$ is up to 6 or more; this reduces the effectiveness of many techniques such as electron diffraction. Dyke, Howard and Klemperer (J. Chem. Phys., 56, 2442 ; 1972), however, found that the hydrogen fluoride from an appropriate supersonic nozzle is cooled sufficiently for appreciable amounts of $(\mathrm{HF})_{2}$ to form, and they obtained satisfactory signals from their molecular beam electric resonance spectrometer using a simple mass spectrometer detection system.

The microwave transitions and the radiofrequency transitions of the Stark effect confirm the non-linear structure with an F ... F distance of $279 \mathrm{pm}$ and favour an angle of $108^{\circ}$ between the HF bonds. Additionally they find an axial component of the electric dipole moment of $10.0 \times 10^{-30} \mathrm{~m} \mathrm{C}(2.99$ debye), and if the central hydrogen atom is replaced by a deuterium atom the nuclear quadrupole coupling is $270 \mathrm{kHz}$. Although signals from F-D . . . FH were easily observed, no signals from $\mathrm{F}-\mathrm{H} \ldots \mathrm{FD}$ were detected, suggesting that this isomer is considerably less stable. For the species $(\mathrm{HF})_{2}$ and $(\mathrm{DF})_{2}$ there was evidence for the exchange of the atom in the centre of the bond so that each hydrogen is there for half the time. The true description of the motion in this degree of freedom is analogous to the inversion mode in ammonia. It can be described by a "tunnel" frequency of about $19.776 \mathrm{GHz}$ in $(\mathrm{HF})_{2}$ but only $1.580 \mathrm{GHz}$ in (DF $)_{2}$ where the effective mass of the "tunnelling" hydrogen is so much larger; a crude estimate of the barrier height is $6 \mathrm{~kJ} \mathrm{~mol}^{-1}$.

The mutual success of these two distinct approaches gives hope for their extension to $\mathrm{H}_{2} \mathrm{OHF}$ and $\left(\mathrm{H}_{2} \mathrm{O}\right)_{2}$ and hence a thorough understanding of hydrogen bonds, at least in the gas phase. The work also confirms that both $a b$ initio calculations, at a level which does not require excessive computer time and sophisticated molecular beam spectroscopy, are powerful tools for difficult structural problems.

\title{
Partons and the Origin of the Universe
}

GeNeral relativity explains the gravitational interaction in terms of fourmotion of particles along geodesics in curved space-time.

An attractive programme is to explain away the other fundamental forces of physics in a similar fashion, and recent work by Wheeler and Misner has made a great deal of progress in this direction. The space-time in Wheeler and Misner's quantum theory is not only curved, but does not even have the familiar topology of Euclidean space. In Wheeler's world fundamental particles exist in worm-holes which are gaps in the space-time continuum.

The latest geometrical theory of fundamental forces seeks to incorporate strong interactions into the geometrical scheme. The present theory, due to Sarasatt, also incorporates the quark model of fundamental particles which provides a symmetrical explanation of the hierarchy of particles, although the prediction that quarks should be observable has not yet been fulfilled.

In an article in next Monday's Nature Physical Science (December 4) which refers to his work to be published in Physical Review Letters, Sarasatt considers the hypothesis that nucleons are made out of more fundamental units, whose structure is geometrical. These units are called partons. Their bare rest mass is enormous, $10^{-5} \mathrm{~g}$, and their radius is $10^{-33} \mathrm{~cm}$. They carry the same spin as the electron and a sixth of the charge. The large mass and small radius come from locating the singular surface which surrounds any massive particle according to general relativity at the worm-hole radius of a particle in Wheeler-Misner theory.

Partons bind together to form mesons and baryons because their gravitational attraction overcomes their electrical repulsion. This binding together takes place in two stages. In the first, three partons bind together to form a quark. Most of their resonance is lost in this stage in the form of emitted energyphotons, gravitational waves and neutrons. In the second stage, quarks bind together to form baryons and mesons according to the SU(3) scheme.

One attractive feature of the theory is that the huge energy release that takes place when partons coalesce might provide a source for the energy emitted from quasi-stellar objects.

It is known that solutions of the equations of classical relativity must contain singularities and such a singularity is the origin of the universe in the big bang model. Sarasatt conjectures that such singularities would split up the partons. This conjecture, like much else in this field, awaits the quantization of general relativity, but it provides a dramatic picture of the creation of the universe by the fusing together of partons to form nuclear matter, releasing encrgy which remains as the "black-body" background. 